\title{
SPACE OBSERVATIONS OF FLARE SPRAYS AND RELATED SURGE PHENOMENA
}

\author{
YNGVE ÖHMAN \\ (Stockholm Observatory, Saltsjöbaden, Sweden)
}

In a publication of the Dunsink Observatory in 1960 (Ellison et al., 1960) the late Dr. Ellison and two of his collaborators presented light curves of 30 solar flares in relation to sudden ionospheric disturbances. Among these flares a solar-limb event of April 22, 1959 was of a particular interest, as it indicated strong X-ray emission from an object situated well outside the limb. This limb flare consisted of a small globule, not particularly bright, which rose $18000 \mathrm{~km}$ in $4 \mathrm{~min}$ and had a total duration of only 5 min. Yet it produced a fine 'sudden enhancement of atmospherics' which persisted for $66 \mathrm{~min}$ after the flare had faded to invisibility.

In my opinion Ellison's fine observation is a good illustration of the fact that our present means for optical recording at ground-based observatories are not always quite satisfactory. A coronagraph with wide band filter may have given a more complete optical recording in this case. And simultaneous X-ray photographs and X-ray spectra would no doubt have been of extremely great interest for a comparison with the optical recording.

In 1960 Kleczek and Křivský (1960) reported some similar observations of sudden enhancement of atmospherics for solar-limb surges. Six such objects not coinciding in time with other $\mathrm{H} \alpha$ events on the Sun gave very clear S.E.A.-effects, interpreted by Kleczek and Krrivský as X-ray radiation emitted by the adjacent region of the solar corona.

With the introduction of direct X-ray recording from satellites the indirectly revealed effects could be controlled and studied in detail. In a paper published in 1962 by Kreplin et al., I quote from the abstract the following clear statement: "Measurements made from the SR-1 satellite confirm the hypothesis that solar X-rays are the cause of flare SID-events. Results also indicate that active prominence regions, bright surges on the limb and certain limb flares have the same $X$-ray characteristics as major disk flares etc."

Among the active prominences listed in this first catalogue of X-ray events was the spectacular object of July 24, 1960. Very good photographs of this prominence were secured by Kerstin Fredga at the Swedish Solar Observatory in Anacapri. Simultaneous photographs were obtained at the German Solar Observatory on the island. These photographs show a twisted structure indicating the presence of magnetic fields.

There have been some contradictory opinions as to the origin of the strong X-ray

Perek (ed.), Highlights of Astronomy, 533-537. (1.A.U. 


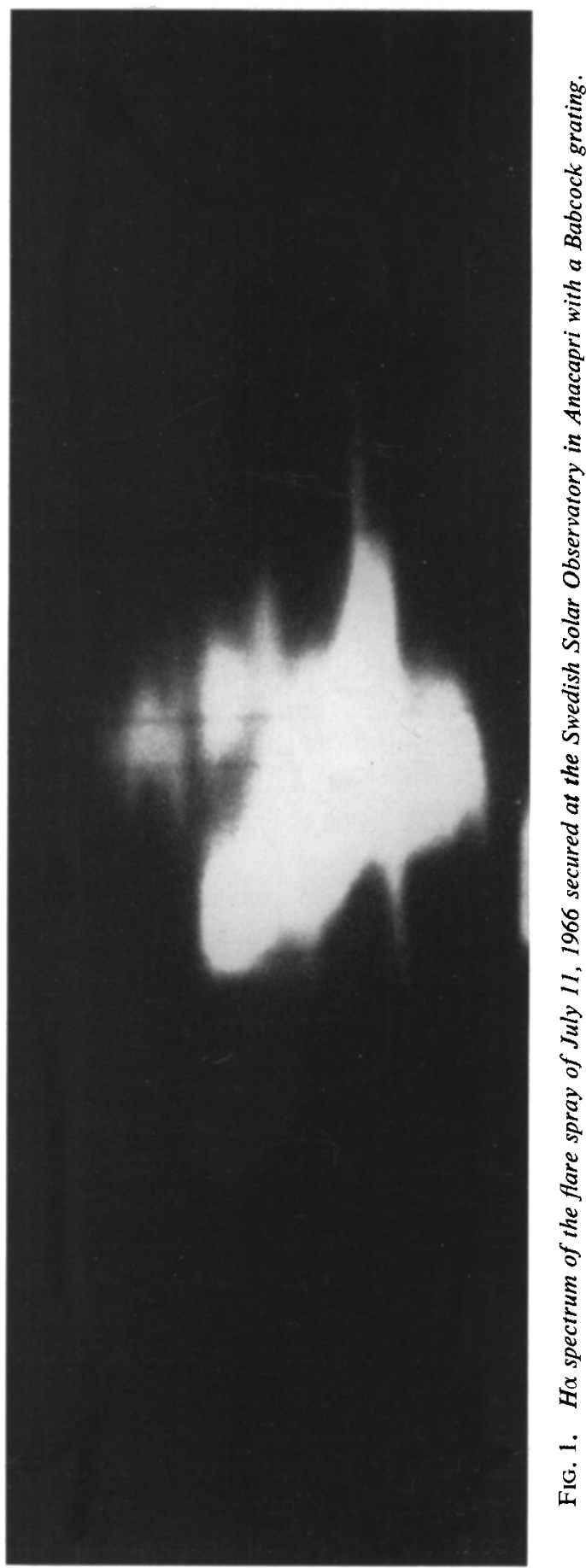


emission below $8 \AA$ accompanying this event. In our opinion the visible surface activity on that occasion could not in itself produce the strong increase in the X-ray flux which was observed. Several similar events have in fact been recorded later on.

A very spectacular one appeared on July 11, 1966 (Öhman et al., 1967). This was a typical flare-spray event. During the brilliant phase the intensity of the object, when measured in our $\mathrm{H} \alpha$ filter in Anacapri, was 1.6 times that of the solar surface near the centre of the disk. Few minutes later the ejected clouds formed a more or less circular or spiral configuration. Because of considerable Doppler velocities the image secured with the $0.7 \AA \mathrm{H} \alpha$ filter gave a rather incomplete recording of the spray.

This is evident when comparing these images with our spectrographic recordings made with a Babcock grating (Figure 1). They show not only a very broad $\mathrm{H} \alpha$ line but also displacements corresponding to velocities of up to about $500 \mathrm{~km}$ per second. A coronagraphic picture obtained with a $5 \AA \mathrm{H} \alpha$ filter $72 \mathrm{~min}$ after the start of the spray still shows a very spectacular prominence, and in spite of the fact that the narrow pass-band filter did not show any clouds at all at this late moment. This shows again the need for a coronagraphic limb-spray patrol, and for improved lineshifters in the solar disk patrol.

The spray of July 11, 1966 appeared suddenly, and perhaps in consequence of a flare situated on the non-visible surface beyond the limb. Sometimes the appearance of such a flare can be inferred from the appearance of bright surges. Very interesting effects of activation have been noted sometimes when such surges penetrate already existing quiescent prominences on the limb. Such an activation was observed in Anacapri on March 25, 1967 (Gimse and Hosinsky, 1967).

Sometimes the sudden activation of a prominence can be followed on the disk not only as a metamorphosis of the filament but as a rapid motion of the whole object as well, and, in fact, in such a way that the filament is transformed to a spectacular ascending prominence far outside the limb. A very fine example of this kind of sudden disappearance was observed by us on June 23, 1967 (Figure 2).

$\mathrm{H} \alpha$ spectra of this object showed great Doppler shifts, but at the same time a fairly narrow line, indicating a slow disintegration process. A very remarkable circularly shaped $\mathrm{H} \alpha$-line picture resulted from some of the structure elements of this object.

We have found similar circular $\mathrm{H} \alpha$ spectra in quiescent prominences too. Some of them show a 'limb brightening', which partly may be due to self-absorption but which may perhaps indicate a rapid rotatory mass motion of filamentary elements as well and, if so, with the axis of rotation more or less parallel to the slit.

Another type of activation of an already existing prominence is characterized not only by Doppler-shifted lines but also by very broad lines suggesting a rapid disintegration. In our opinion this type of activation is more likely to be accompanied by $\mathrm{X}$-ray emission than the one characterized by narrow spectral lines.

In connection with the tentative suggestion that rotatory mass motions may appear in prominences I want to take this opportunity to mention that somewhat similar 

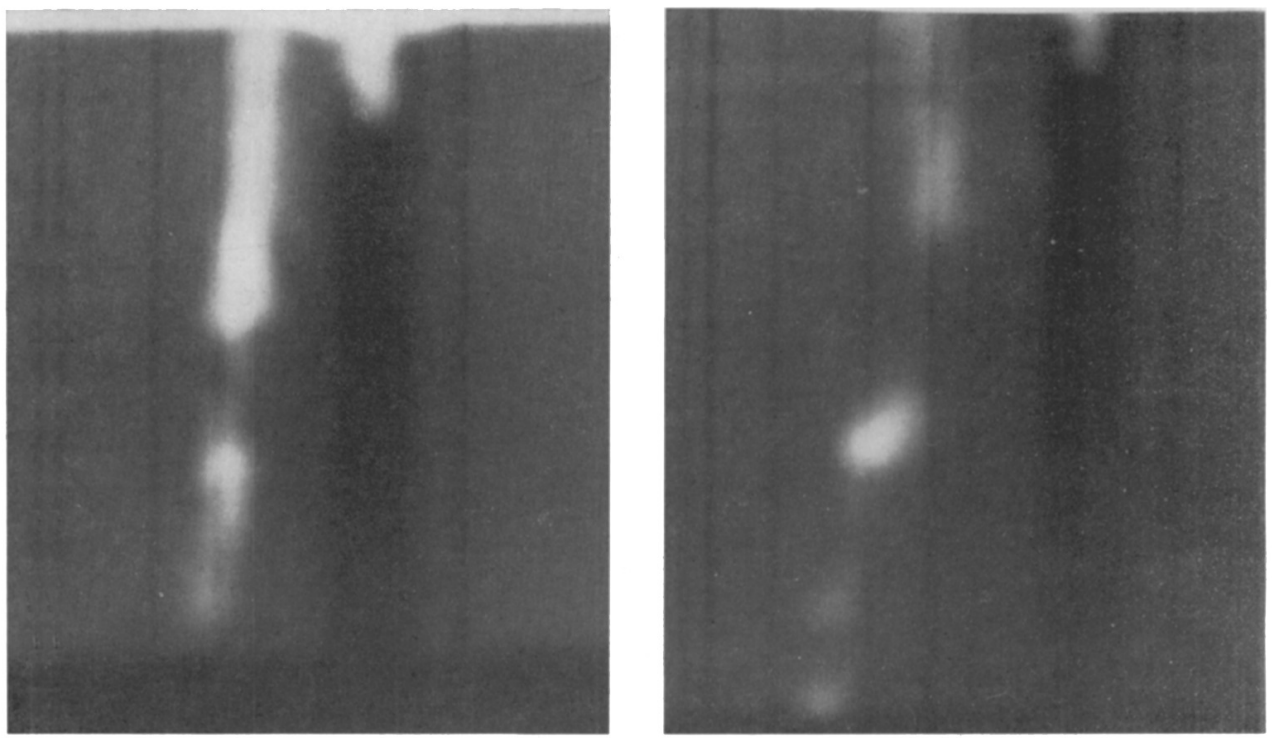

FIG. 2. Ha spectra of a sudden disappearance observed in Anacapri on June 23, 1967 at UT 13:16

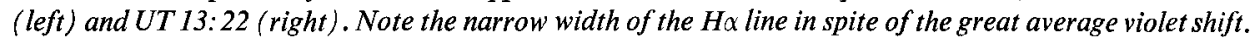

phenomena have been found by us in solar flares too. In this case the rotation of the gases is indicated by a slightly inclined $\mathrm{H} \alpha$ streak. The axis of rotation would in such a case be more or less perpendicular to the slit. In private conversations with Professor Alfvén, I have been informed that a rotation may well be expected according to his and Carlqvist's recent theory of solar flares (1967). Reference should also be given to an interesting paper by Maria Cristina Ballario, who has discussed the possibility that complex Doppler shifts may appear in flares if these have a loop structure and with rapid mass motion (Ballario, 1963).

But can such a rotation of structure elements in say flares, surges, spicules or prominences be of any interest to space research and X-ray studies in particular? In my opinion this may well be the case. In a recent paper Kuperus and Tandberg-Hanssen (1967) have examined in detail how the filamentary structures of prominences may be thermally insulated from the hot corona. It seems to me that a rotation is likely to produce a greater leakage of gas. Even if such a phenomenon may produce only a minor thermal increase in X-ray emission and no bursts of the type appearing in connection with explosive events such as surges and sprays we may have an effect worth studying.

The extremely good X-ray pictures secured on November 12, 1966 by Underwood and Mune have shown to us that fairly small details can already be studied on X-ray solar photographs (Underwood and Mune, 1967). It is important therefore that the methods in optical observation are developed so as to take advantage of this rapid 
development of X-ray solar research. Similar requirements may be made from microwave solar astronomy, particularly because of the intimate connection between the burst phenomena observed in X-rays and microwaves.

We have considered here mainly one branch of space research, the X-ray astronomy. Needless to say such research as ultraviolet spectroscopy etc. would certainly be of a great interest in connection with flare sprays too. With the good progress made recently in the study of the solar limb by Wilson and others (Burton et al., 1967) such spectroscopic studies seem well possible.

Even such a difficult task as observing say the Lyman continuum in such objects seems possible. If so, a monochromator developed by us recently by the use of subtractive dispersion (Öhman, 1967) may perhaps find applications. With two gratings the spectrum can be made to turn, and at the turning-point a sharp image of say a spray flare can be produced in any selected wavelength range of its continuous spectrum.

\section{References}

Alfvén, H., Carlqvist, P. (1967) Solar Phys., 1, 220.

Ballario, M.C. (1963) Osservatorio Arcetri, Contr., 78.

Burton, W. M., Ridgeley, A., Wilson, R. (1967) Mon. Not. R. astr. Soc., 135, 207.

Ellison, M.A., McKenna, Susan, M.P., Reid, J.H. (1960) Cape Lyot Heliograph Results No. 1.

Gimse, O., Hosinsky, G. (1967) Solar Phys., 2, 192.

Kleczek, J., Křivský, L. (1960) Nature, 186, 1035.

Kreplin, R.W., Chubb, T.A., Friedman, H. (1962) J. geophys. Res., 67, 2231.

Kuperus, M., Tandberg-Hanssen, E. (1967) Solar Phys., 2, 39.

Öhman, Y. (1967) Nature, 215, 606.

Öhman, Y., Stiber, G., Kusoffsky, U. (1967) Solar Phys., 1, 60.

Underwood, J.H., Muney, W.S. (1967) Solar Phys., 1, 129. 